\title{
Ecological niche models reveal the importance of climate variability for the biogeography of protosteloid amoebae
}

\author{
María Aguilar and Carlos Lado \\ Mycology Department, Real Jardín Botánico, CSIC, Plaza de Murillo 2, Madrid, Spain
}

\begin{abstract}
Habitat availability and environmental preferences of species are among the most important factors in determining the success of dispersal processes and therefore in shaping the distribution of protists. We explored the differences in fundamental niches and potential distributions of an ecological guild of slime moulds-protosteloid amoebae-in the Iberian Peninsula. A large set of samples collected in a north-east to south-west transect of approximately $1000 \mathrm{~km}$ along the peninsula was used to test the hypothesis that, together with the existence of suitable microhabitats, climate conditions may determine the probability of survival of species. Although protosteloid amoebae share similar morphologies and life history strategies, canonical correspondence analyses showed that they have varied ecological optima, and that climate conditions have an important effect in niche differentiation. Maxent environmental niche models provided consistent predictions of the probability of presence of the species based on climate data, and they were used to generate maps of potential distribution in an 'everything is everywhere' scenario. The most important climatic factors were, in both analyses, variables that measure changes in conditions throughout the year, confirming that the alternation of fruiting bodies, cysts and amoeboid stages in the life cycles of protosteloid amoebae constitutes an advantage for surviving in a changing environment. Microhabitat affinity seems to be influenced by climatic conditions, which suggests that the micro-environment may vary at a local scale and change together with the external climate at a larger scale.
\end{abstract}

The ISME Journal (2012) 6, 1506-1514; doi:10.1038/ismej.2012.12; published online 8 March 2012

Subject Category: microbial population and community ecology

Keywords: distribution; ecogeography; protists; protostelids; slime moulds

\section{Introduction}

General biogeographic patterns of free-living protists are still a subject of debate. The 'everything is everywhere' hypothesis states that most free-living protists have huge population numbers and a small body size, which may cause high rates of dispersal and a low rate of allopatric speciation and endemism (Finlay and Clarke, 1999; Finlay et al., 1999, 2001; Finlay, 2002; Finlay and Fenchel, 2004). Therefore, the individual environmental preferences of the species and habitat availability would be major forces in shaping their distributions (Finlay, 2002; Fenchel and Finlay, 2006). On the other hand, there is also evidence of limited dispersion and geographically restricted organisms (Foissner, 2006; Smith and Wilkinson, 2007; Foissner et al., 2008; Vanormelingen et al., 2008), which are consistent with a 'moderate endemicity' scenario.

Correspondence: M Aguilar, Mycology Department, Real Jardín Botánico, CSIC, Plaza de Murillo 2, Madrid 28014, Spain.

E-mail: aguilar@rjb.csic.es

Received 3 November 2011; revised 24 January 2012; accepted 24 January 2012; published online 8 March 2012
The fundamental niche of a species is the set of environmental conditions that make possible its long-term survival (Hutchinson, 1957), excluding the effect of biotic interactions, restricted dispersion, or human influence, that can prevent the species from inhabiting all the areas encompassing its full ecological potential (Pulliam, 2000; Anderson and Martínez-Meyer, 2004). Using ecological niche modelling techniques, it is possible to devise a model of a species' environmental requirements from the conditions of sites of known occurrence, obtaining a mathematical function that represents its fundamental niche. Results can later be projected into new areas with known characteristics to predict the probability of presence of the species there and trace their potential distributions (Phillips et al., 2006).

In this paper, we explore the differences in fundamental niches and potential distributions of protosteloid amoebae in an area of the south-west of Europe, the Iberian Peninsula. Protosteloid amoebae, formerly known as protostelids, constitute an ecological guild of slime moulds that are scattered within the amoebozoa tree (Shadwick et al., 2009b, Supplementary Figure S1), and act as predators of 
decomposers of decaying plant tissues (Spiegel, 1986; Spiegel et al., 2007). All protostelid species have in common that they are amoeboid organisms with the ability, under certain conditions, to produce microscopic fruiting bodies (Olive, 1975; Spiegel, 1986; Spiegel et al., 2007), which consist on one to a few spores at the tip of a delicate acellular stalk. They also produce various trophic stages that range from amoebae or amoeboflagellates to microscopic plasmodia. Available data on the ecology and distribution of these organisms show evidence that compositional differences exist between microhabitat types at a local scale (Moore et al., 2000; Moore and Spiegel, 2000b, 2000c; Shadwick and Stephenson, 2004; Powers and Stephenson, 2006; Aguilar et al., 2007; Kosheleva et al., 2009). Microhabitats are small, localised habitats within a larger ecosystem and have their own environmental characteristics that presumably are more or less constant across areas with similar climates. On a continental scale, is it also possible to find different communities in the same microhabitat, caused by different climate conditions (Ndiritu et al., 2009; Aguilar et al., 2011). Thought not statistically tested, there is a tendency that at least some species move towards ground litter in boreal areas (Spiegel and Stephenson, 2000), and towards aerial litter in tropical areas (Moore and Spiegel, 2000c).

Approximately $80 \%$ of the surface of the Iberian Peninsula has a Mediterranean climate. This climate is characterized by warm to hot, dry summers and mild to cool, wet winters. There is always a summer drought caused by subtropical high-pressure cells, that make rainfall very unlikely except for occasional thunderstorms, and almost all precipitation falls during the colder months of the year. In Mediterranean regions that are in the proximity of the sea, temperatures are generally moderate with a comparatively small annual temperature range. Regions further from the coastal areas have a lower temperature in the winter and high annual temperature ranges (Di Castri et al., 1981; Walter, 1984; Agencia Estatal de Meteorología, 2011). On the other hand, areas with an Oceanic climate, that cover the remaining $20 \%$ of the peninsular area and are located on the northern coastal strip, have moderately cool summers and warmer winters than in the inland areas, with a narrow annual temperature range. They lack a dry season and precipitation is evenly dispersed through the year.

The objective of this study was to explain the geographical patterns of protosteloid amoebae in the Iberian Peninsula from an ecological point of view. For this, we used a large number of samples collected along a Northeast to Southwest diagonal transect, with comparable methodology. Maxent environmental niche models and canonical correspondence analyses (CCA) were used to evaluate the hypothesis that ecological niches of protosteloid species strongly collaborate in shaping their geographic distribution, and that once their ecological preferences are known, it is possible to predict their probability of occurrence in other similar areas not yet studied. Both climatic variables and microhabitat were included in CCA, in order to evaluate and compare their effects.

\section{Materials and methods}

\section{Sampling}

Results presented in this study were obtained by the analysis of data collected in the Iberian Peninsula during years 2005-2009, along a diagonal transect of approximately $1000 \mathrm{~km}$ from the Northeast to the Southwest of the peninsula. At each site, samples were collected within a radius of approximately $20 \mathrm{~m}$. We aimed to collect 10 samples from different plant species in each locality for subsequent laboratory culture, but it was not possible in all cases due to the absence of suitable tissues. The 10 samples belonged to different microhabitats and were distributed as follows: 4 samples of aerial litter (assemblage of dead but still attached parts of standing plants), 4 samples of ground litter (the layer of twigs, leaves and other plant debris extending over the soil surface), and 2 samples of bark of living plants. Collected samples were placed in individual paper bags and air-dried in the laboratory. They were cultured as described elsewhere (Aguilar et al., 2011), and identified on the basis of fruiting body morphology. Species abundances were quantified following the methods based on colony counts described in Aguilar et al. (2011).

\section{Database}

The number of colonies from each species in each sample was recorded in a database, also containing microhabitat type and spatial coordinates of the localities. To improve the quality of the models, data already published in Aguilar et al. (2007, 2011) were also included. This database was refined to avoid excessive differences in sampling effort that may bias the results. Sampling sites were projected on a geographic information system (GIS), and points were randomly eliminated from oversampled areas. The points finally selected (Figure 1) were associated with data from a total of 23 species. With this database already refined, two matrices were built, for use in the analyses later. The first matrix contained presence data of the species that were present in at least 10 localities (Supplementary Table S2). The second matrix included abundance data of species in different microhabitats at each site (Supplementary Table S3), but as in some cases the samples were cultured more than once (see Aguilar et al., 2011), culturing effort differences in samples were corrected by dividing the number of colonies between the number of cultures and rounding down results. A third matrix was constructed with values 


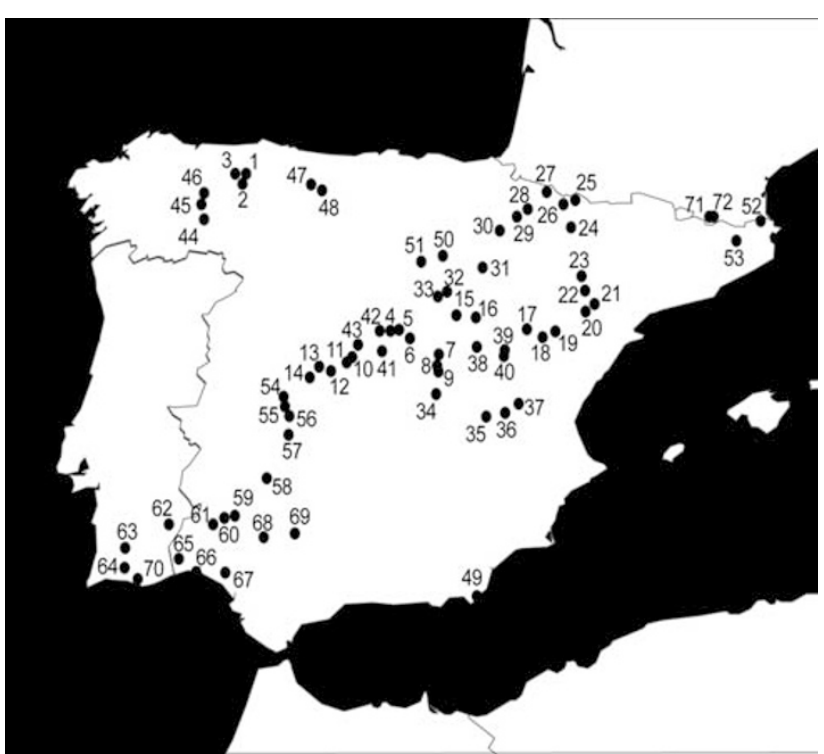

Figure 1 Map of the Iberian Peninsula with selected localities for the analyses. Localities are represented as circles, and numbers correspond to information in Supplementary Tables S3 and S4.

of the 19 Bioclim variables from the WorldClim database (Hijmans et al., 2005) (www.worldclim.org, March 2011) in current conditions with a 30 arcseconds resolution for each selected locality, that were extracted with Spatial Analyst extension of ArcGIS (Supplementary Table S4).

\section{Maxent}

The matrix with presence data was analysed with the program Maxent version 3.3.3e (freely available at http://www.cs.princeton.edu/ schapire/maxent/), March 2011 (Phillips et al., 2006; Phillips and Dudik, 2008). Niche models for the species present in at least 10 localities (14 species) were calculated with Bioclim variables from WorldClim in current conditions with a 30 arc-seconds resolution. Preliminary models were developed using all 19 variables. To prevent over-fitting, the variables that were considered to contribute less to the model were removed after observing the estimates of their relative contributions, and the jacknife tests of variable importance implemented in the Maxent software. Finally, the models were run with selected variables only, and with $80 \%$ of the occurrence localities as training data, reserving the remaining $20 \%$ for testing results. Models were evaluated based on receiver operating characteristic analysis that generates the AUC (area under the curve) score.

\section{Canonical correspondence analyses}

Correlation between all pairs of climatic variables in the matrix with extracted values from Bioclim at each point, was studied using regression analyses in
R 2.12.2 (R Development Core Team, 2008) (Supplementary Table S5), and highly correlated variables without a clear biological significance in the study area considered-mean temperature of the warmest quarter, precipitation of the driest month, precipitation of the wettest quarter and precipitation of the driest quarter-were removed. A stepwise CCA was performed with $\mathrm{R} 2.12 .2$ and the vegan package (Oksanen et al., 2008). This analysis sequentially removes the least important variables, and thus makes possible to distinguish which variables contribute more to differentiate the niches of the species. Species were scaled proportional to eigenvalues, sites were unscaled (weighted dispersion equal on all dimensions), and permutation tests were carried out.

\section{Mantel tests}

Geographic distances between sampling points, and Bray-Curtis dissimilarity between assemblages were measured using the packages fields (Nychka, 2007) and ecodist (Goslee and Urban, 2007) in R. Ecological distance between localities was calculated as an Euclidean distance in a multidimensional space determined by the 19 Bioclim variables previously centered and scaled. Mantel tests and partial mantel tests were performed with vegan, using 1000 permutations, and Pearson product-moment correlation coefficient.

\section{Results}

\section{Maxent}

Variables selected for each species model and their relative contributions are shown in Table 1. All models had high AUC scores ( $>0.9$ ) for both training and test data. Environmental variables that most frequently had a high percentage contribution to the models were isothermality, mean diurnal range, precipitation of the coldest quarter, temperature seasonality, precipitation of the warmest quarter, and precipitation seasonality.

Projected models are displayed in Figure 2. Most species prefer the inland areas of the northern half of the peninsula and the eastern coastal strip. All these areas are characterized by low annual precipitation (generally $<600 \mathrm{~mm}$ ) and low precipitations even in the winter (ranging 65-145 $\mathrm{mm}$ in the coldest quarter of the year), but higher precipitations in the summer than in other Mediterranean areas of the peninsula (50-100 $\mathrm{mm}$ ). Temperatures are relatively cold (annual means ranging 9.5-14.5) and vary throughout the year (s.d. 5.5-6.7).

But some species, namely Protostelium mycophagum, Endostelium zonatum, Nematostelium ovatum and Protostelium pyriforme, can also tolerate areas in the Southwest, with higher annual mean temperatures $\left(14-18{ }^{\circ} \mathrm{C}\right)$ and a lower temperature seasonality (s.d. 3.6-5.2). These areas are also characterized by a 
Table 1 Results of the maxent environmental niche models

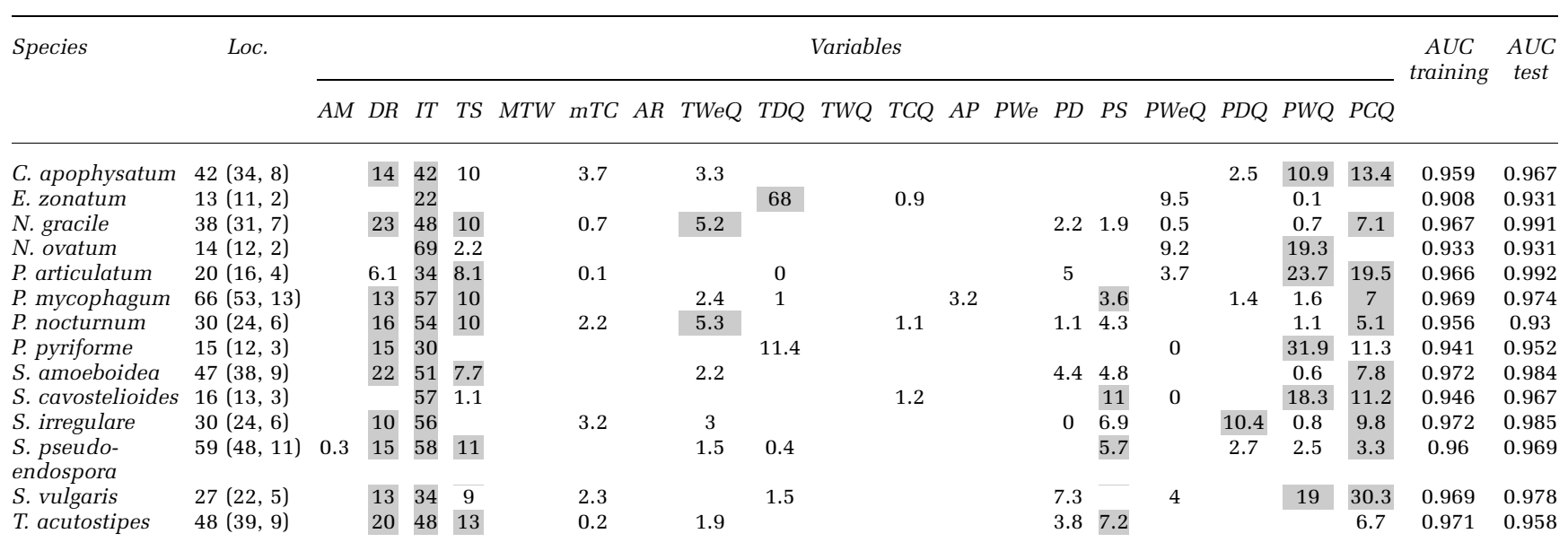

Abbreviations: Loc., total number of localities (training, test); AM, annual mean temperature; AP, annual precipitation; AR, temperature annual range; AUC test, area under the ROC curve for test data; AUC training, area under the ROC curve for training data, DR, mean diurnal range; IT, isothermality; mTC, minimum temperature of the coldest month; MTW, maximum temperature of the warmest month; PCQ, precipitation of the coldest quarter; PD, precipitation of the driest month; PDQ, precipitation of the driest quarter; PS, precipitation seasonality; PWe, precipitation of the wettest month; PWeQ, precipitation of the wettest quarter; PWQ, precipitation of the warmest quarter; TCQ, mean temperature of the coldest quarter; TDQ, mean temperature of the driest quarter; TS, temperature seasonality; TWeQ, mean temperature of the wettest quarter; TWQ, mean temperature of the warmest quarter.

The table gives the estimated percentage of relative contribution of selected Bioclim variables for each species' final model, and the area under the receiver operating characteristic (ROC) curve for training and test data. The $50 \%$ variables with higher contributions for each species are highlighted in grey.

higher precipitation in the winter than in other Mediterranean areas in the Iberian Peninsula (150$250 \mathrm{~mm}$ in the coldest quarter of the year), and a severe summer drought (precipitation $<50 \mathrm{~mm}$ in the warmest quarter of the year). Some other species, like Schizoplasmodiopsis vulgaris, Schizoplasmodiopsis pseudoendospora, Protosporangium articulatum, Soliformovum irregulare and Cavostelium apophysatum, have large areas predicted with very high probability $(0.8-0.9)$ in the north-east of the peninsula, in an area with very low annual precipitation $(140-160 \mathrm{~mm})$, and low precipitation both in colder $(<70 \mathrm{~mm})$ and warmer months $(70$ $100 \mathrm{~mm}$ ), moderately high annual mean temperature $\left(13-16{ }^{\circ} \mathrm{C}\right)$, with relatively high temperature seasonality (s.d. 6-6.5).

\section{Canonical correspondence analyses}

After the stepwise process, the independent variables that were not removed by the analysis and were used to generate the final ordination, were Bioclim's annual mean temperature, isothermality, precipitation seasonality, precipitation of warmest quarter, precipitation of coldest quarter, aerial litter microhabitat, ground litter microhabitat, and bark microhabitat.

The final CCA obtained (Figure 3) had a total inertia of 3.1527 , a constrained inertia of 0.5522 (proportion $17.52 \%$ ), and an unconstrained inertia of $2.6005(82.48 \%)$. The permutation test for the axes was significant $(P=0.005)$, and the permutation test for the independent variables showed that isothermality $(P=0.010)$, precipitation seasonality $(P=0.055)$, precipitation of warmest quarter
$(P=0.005)$, precipitation of coldest quarter $(P=0.005)$, aerial litter microhabitat $(P=0.005)$, and ground litter microhabitat $(P=0.080)$, and bark microhabitat $(P=0.05)$ had significant effects.

The species that have preference for ground litter and bark microhabitats in this areas-S. pseudoendospora, N. ovatum, Nematostelium gracile, Schizoplasmodium cavostelioides, Schizoplasmodiopsis reticulata, Endostelium amerosporum, C. apophysatum and Schizoplasmodiopsis amoeboidea-are more abundant where annual mean temperature is high, and precipitation of the coldest quarter is low. On the other hand, the species that typically inhabit aerial litter here-P. mycophagum, P. pyriforme and Protosporangium bisporum-are more frequently identified in localities with lower annual mean temperature, and higher precipitation seasonality.

There is a group of species with affinities for areas with high isothermality and precipitation seasonality but low precipitations both in colder and warmer months-Tychosporium acutostipes, S. irregulare, P. articulatuim and $S$. vulgaris. By contrast, another group of species shows clear preference for high precipitation of the warmest quarter values and low precipitation seasonality and isothermalityMicroglomus paxillus, Protostelium nocturnum, Echinostelium bisporum, Echinosteliopsis oligospora, E. zonatum and Protostelium arachisporum.

\section{Mantel tests}

Bray-Curtis dissimilarities between protosteloid assemblages were less correlated with geographic 

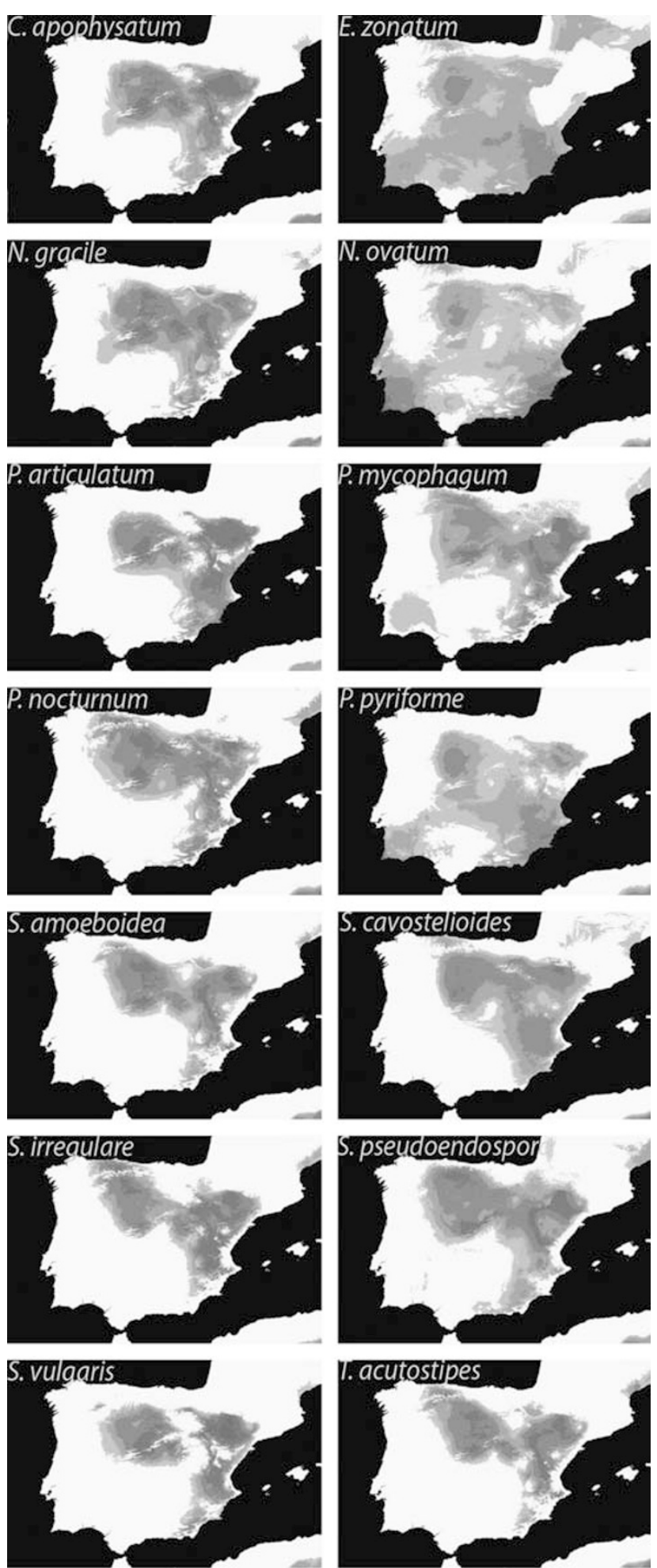

1. acutostipes

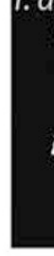

LOGISTIC OUTPUT

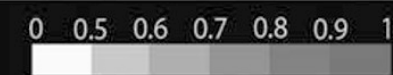

Figure 2 Maxent predictive ecological models of protosteloid amoebae with more than 10 occurrences. Probabilities of presence $>0.5$ are represented using different shades of grey.

distance $(r=0.1464, P=0.003)$, than with ecological distance $(r=0.2453, P<0.001)$. Using partial mantel tests, Bray-Curtis dissimilarities were even less

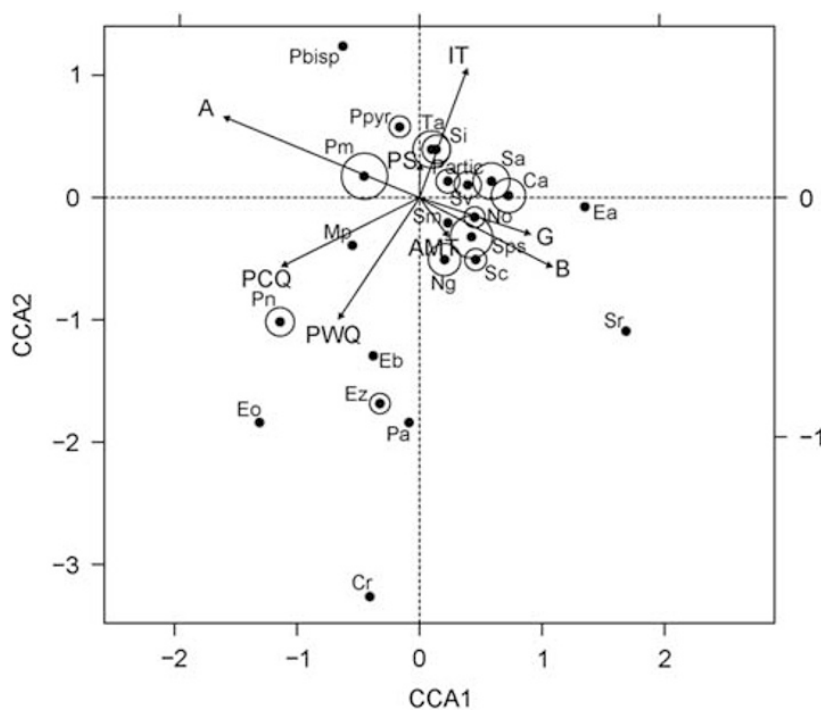

Figure 3 CCA using species as dependent variables and climatic and microhabitat variables as independent variables. Each species point in the diagram is at the centroid (weighted average) of the sites in which it occurs. Diameters of circles around the points are proportional to the logarithm of the species' absolute abundances. Environmental variables are represented by arrows that run from the origin to the weights that each variable has in the linear combinations that form the axes. A, aerial litter; G, ground litter; B, bark; AM, annual mean temperature; IT, isothermality; PS, precipitation seasonality; PCQ, precipitation of the coldest quarter; PWQ, precipitation of the warmest quarter; Ca, $C$. apophysatum; Cr, Clastostelium recurvatum; Ea, E. amerosporum; Eb, E. bisporum; Eo, E. oligospora; Ez, E. zonatum; Mp, M.paxillus; Ng, $N$. gracile; No, N. ovatum; Partic, P. articulatum; Pbisp, P. bisporum; Pa, P. arachisporum; Pm, P. mycophagum; Pn, $P$. nocturnum; Ppyr, P. pyriforme; Sa, S. amoeboidea; Sm, S. micropunctata; Sps, S. pseudoendospora; Sr, S. reticulata; Sv, S. vulgaris; Sc, S. cavostelioides; Si, S. irregulare; Ta, T. acutostipes.

correlated with geographic distance when removing the effect of ecology $(r=0.0291, P=0.256)$. On the other hand, the correlation between Bray-Curtis dissimilarities and ecological distances did not significantly decrease after removing the effects of geographic distance $(r=0.201, P<0.001)$.

\section{Discussion}

How does the environment select? Modelling fundamental niches

A better understanding of the ecology and dispersal mechanisms of protists is essential for interpreting their biogeographical patterns. In small organisms with an efficient dispersion, the availability of an appropriate habitat would be the principal filter for their establishment on a new area. Knowing the requirements of a species it would be possible to check if propagules can reach all potentially suitable habitats or, on the contrary, their dispersion has been limited in some directions.

The size range of protostelid spores (ca. 4-50 $\mu \mathrm{m}$ in diameter) gives them the potentiality to be easily dispersed, which justifies to use the 'everything is 
everywhere' model as an approximation to what is happening to these organisms. Assuming that the distribution of the species is not hindered by geographic constraints, as the results of the Mantel tests confirm, the probable distribution of the species was extrapolated based on the climatic data of the sites where they were found.

When constructing predictive models of the niche of a species, the goal is to predict which areas form part of its potential distribution (Anderson and Martínez-Meyer, 2004). To create a satisfactory model, it is very important to make a cautious sampling design to get a sufficiently representative sample. Our sampling design attempted to balance three objectives. One objective was to cover a wide sampling area with different climates to have a bigger picture of the species' ecology, and also to address the dispersal efficiency of protostelids at this scale. Another was to know each locality in sufficient detail. And finally, the third objective was to equalise the effort along the whole transect. But for interpreting the results, we must keep in mind the limitations of our models. As a consequence of the sampling strategy, the generated models do not represent predictions of presence/absence of the species in absolute terms, but provide an estimate of the probability of finding protosteloid amoeba in Mediterranean areas of the Iberian Peninsula using the same methodology and effort.

Our results show that, despite that protosteloid amoebae are considered to share similar morphological characteristics and life history strategies, their environmental niches are not completely the same and each species has its own climatic and microhabitat preferences, confirming results obtained in Aguilar et al. (2011) with a smaller data set. Differences in climatic conditions cause the species composition and the structure of the assemblages to vary from locality to locality, being this influence stronger than the effects of geographic distance. These results are also supported by previous studies, which, although do not deal with the influence of climatic variables on protostelid assemblages, show that there are differences in species composition when comparing areas with temperate (Best and Spiegel, 1984; Moore and Spiegel, 1995, 2000a, 2000b; Shadwick and Stephenson, 2004; Tesmer et al., 2005; Aguilar et al., 2007; Brown and Spiegel, 2008; Shadwick et al., 2009a), tropical (Stephenson et al., 1999; Moore and Spiegel, 2000c; Moore and Stephenson, 2003; Powers and Stephenson, 2006; Ndiritu et al., 2009), and boreal climates (Moore et al., 2000; Spiegel and Stephenson, 2000; Kosheleva et al., 2009).

\section{Influence of the climate}

For the elaboration of the Maxent models and CCA all the Bioclim variables were initially included, but final models were constructed after removing less informative climatic factors. All 19 Bioclim variables were considered in preliminary Maxent models, and the least important were subsequently removed to prevent possible over-fitting artifacts. For the CCA, preliminary pairwise regression analyses were used to evaluate correlation and to remove highly redundant variables that were considered as less informative in a Mediterranean/ Oceanic climate. After that, the analysis was run in several steps, which sequentially removed the variables with a lower contribution. It is remarkable that the climatic factors selected were in both cases variables that measure changes in conditions throughout the year, like isothermality, temperature ranges, precipitation seasonality, and precipitation of the coldest and warmest quarters. Protosteloid amoebae seem to have the ability to resist variations in their environment, as their peculiar morphology suggests. Spores can survive for a long time and during prolonged periods of drought (Kosheleva et al., 2009), and their life cycles, that alternate stalked fruiting bodies with trophic stages that vary from amoeboflagellates to nonflagellated amoebae, reticulate plasmodia, and cysts (Olive, 1975; Spiegel, 1986), seem particularly suited to make possible their survival with changing external conditions. The CCA results (Figure 3) suggest that what differentiates the climatic niches of the species is precisely the type of change-temperature, precipitation or both- and the magnitude of change that can be tolerated by each of them.

Unfortunately, previous information about the ecology of these organisms is scarce and all available data have been obtained with various methodologies that are not necessarily comparable. Also, results obtained in this study cannot necessarily be extrapolated to a worldwide scenario, and tendencies may be different in other major climates. Therefore, comparisons between studies must be made with caution. Maxent, based on known presence data, predicts the ecological conditions in which it is likely that a species can survive, and CCA uses abundance data to represent the species ecological optima as points in a new coordinate system which maximises their differences. Thus Maxent models give us the opportunity to compare the similarities between species, whereas CCA is a powerful tool to analyse their differences.

According to Maxent models (Figure 2), most protosteloid species can be easily found in inland areas located in the northern half of the Iberian Peninsula and the eastern coast, with mild annual mean temperatures, a moderate annual temperature range and milder drought periods. But some species show also a greater tolerance for more extreme variation. A group of species-P. mycophagum, $E$. zonatum, $N$. ovatum and $P$. pyriforme-has a higher tolerance to areas with higher temperatures with little variation along the year, and a lower precipitation in summer. This species seem to be evolutionary unrelated (Spiegel, 1986; Shadwick et al., 2009b, Supplementary Figure S1), and little is known about their ecology in other areas. Most of 
them have been reported to show preferences for tropical and temperate areas. P. mycophagum, is usually a very abundant species worldwide, and it is probably a generalist with a wide niche (Spiegel et al., 2007; Ndiritu et al., 2009; Aguilar et al., 2011). By contrast, E. zonatum is usually occasional or rare, and it tends to be more common in tropical areas, frequently found on substrates collected in relatively dry habitat and exposed to direct sunlight (Spiegel et al., 2007), and has not been found in boreal climates (Moore et al., 2000; Spiegel and Stephenson, 2000; Kosheleva et al., 2009). N. ovatum is one of the most common species in samples from the lowland tropics but it is also frequent in temperate areas (Spiegel et al., 2007). Finally, P. pyriforme is more abundant in the tropics than in temperate areas (Spiegel et al., 2007).

In the CCA (Figure 3) a group of species, most of them with unknown evolutionary affinities (Supplementary Figure S1), showed the tendency to be more abundant in localities with relatively high precipitation and low seasonal changes of temperature and precipitation, and at least some of them were also common in localities with high temperatures. M. paxillus, E. bisporum, E. oligospora, $E$. zonatum and $P$. arachisporum are species usually rare in temperate climates and very rare in higher latitudes, but usually more abundant in tropical localities (Spiegel et al., 2007; Ndiritu et al., 2009). On the other hand, $P$. nocturnum is more abundant in studies from temperate areas (Ndiritu et al., 2009), and also showed affinity for high precipitation in Aguilar et al. (2011).

Comparing results from Maxent and CCA, it seems that a group of species has high probability of occurrence in dry areas, usually warm and with moderate to high isothermality. One of them is $S$. vulgaris, which according to previous data seems to be more common in temperate areas than in tropical or boreal climates (Ndiritu et al., 2009), and it can also be survive in cool, moist habitats (Spiegel et al., 2007). However, S. pseudoendospora is a very abundant species in most localities studied around the world, especially in temperate and tropical areas (Spiegel et al., 2007), and it also showed preference for warmer temperatures in Aguilar et al. (2011). $P$. articulatum, is a typical inhabitant of bark, so there is little data on it, because this microhabitat is often understudied. However, it has been abundant in some temperate (Ndiritu et al., 2009) and boreal areas (Kosheleva et al., 2009). It appears to be a species that is often associated with arid habitats, and it can occur at higher elevations $(>3000 \mathrm{~m})$ than most protostelids (Spiegel et al., 2007). A species that is common worldwide but seems to be more frequent in temperate areas (Spiegel et al., 2007; Ndiritu et al., 2009), S. irregulare, showed preference for higher precipitations and lower temperatures in Aguilar et al. (2011). C. apophysatum is found more frequently in tropical areas (Spiegel et al., 2007). However, T. acutostipes does not have a clear latitudinal pattern, but it preferred higher temperatures in Aguilar et al. (2011).

\section{Interaction of climate and microhabitat}

Microhabitat type also had an important influence on niche segregation, and it has been frequently mentioned as a very important factor in the ecology of these organisms (Olive, 1975; Spiegel, 1986; Spiegel et al., 2004). When studying a locality in detail, species assemblages from each microhabitat frequently differ more than assemblages from the same microhabitat in nearby localities (Moore and Spiegel, 2000b; Spiegel et al., 2004). However, on a continental scale, species composition may vary in each microhabitat at different latitudes (Ndiritu et al., 2009). On the scale of this study, the effect of microhabitats is strong, but it is not known whether their influence is determined by characteristics of the microhabitats themselves-chemical composition, $\mathrm{pH}$, decomposing stage and so on-or by the assemblage of other interacting organisms that the microhabitats can harbour (Spiegel, 1986). With the methods employed in present research, it is not possible to know in detail the characteristics of each microhabitat type, but they were included in the analyses to get a glimpse of their overall influence on each species, awaiting further characterization in the future.

Results from CCA (Figure 3) show that in the Iberian Peninsula there is a correlation between microhabitat affinity and preference for certain climatic conditions. This correlation is not as strong as when using a more limited set of localities (Aguilar et al., 2011), probably due to the effect of other variables not incorporated in the analyses. With these new results it is possible, however, to visualise the global microhabitat affinity of the species at these latitudes. A tendency appears that typical ground-litter and bark inhabitants prefer higher temperatures and lower precipitation in winter than the species that appear more frequently in aerial litter, which in turn can tolerate higher precipitation seasonality. As most species considered in this study can survive in at least two different microhabitats, it is also possible that their climatic optima vary in each microhabitat. Clarifying these patterns of interaction between microhabitats and climate is essential for understanding the biogeography of protosteloid amoebae because differential microhabitat selectivity could be a strategy for increasing protostelid ability to tolerate larger climatic and geographic ranges.

\section{Future directions and conclusions}

Species' fundamental niche models, and studies of niche selection can become a very useful tool in the future of protist biogeography. Hypotheses related to the ubiquity of protists' dispersal and its equiprobability in all directions (see Foissner, 2006; Weisse, 
2008) deal with the probability of an organism to be transported between suitable habitats, and thus can not be easily falsifiable without an adequate knowledge of the species' ecology. Niche models can be used to generate null hypotheses for an 'everything is everywhere' scenario, that is, they make it possible to identify potential high-probability areas and check for the actual presence of the organisms at both sides of a hypothetical barrier.

It has been demonstrated that climate, and other ecological factors interact with diversity to drive macroevolutionary dynamics (Ezard et al., 2011). The comparison of niches of groups of closely related species can also allow us to determine whether niche differentiation has had an important role in their diversification. Niche segregation based on differences in microhabitat and/or tolerated climate ranges may have been a strategy for avoiding high niche overlap and competitive exclusion in cooccurring species, thus permitting coexistence of organisms that compete for the same resources (Pianka, 1974). In this context, a situation that seems to be more common than previously expected in protists and has not been investigated in protostelids yet, is the existence of morphospecies constituted by complexes of cryptic species, which may have distinct ecological preferences and distributions (Amato et al., 2007; Smirnov, 2007; Morard et al., 2009; Douglas et al., 2011). Knowing in more detail the fundamental and realized niches of ecologically similar species can give us new data for analysing all these processes.

In conclusion, the distribution of protosteloid amoebae in the Iberian Peninsula is not random nor spatially autocorrelated, but it is determined by the niche of each organism and the availability of habitats necessary for their survival. Although they share many morphological similarities and have common habitats, each species has its own ecological preferences, determined by their climatic optima and microhabitat colonisation capacity. As revealed in this study, the effect of microhabitats is strong and comparable with the effects of climate at the scale of the Iberian Peninsula, but it is not known whether the influence of the microhabitat is due to biotic or abiotic factors, and needs further investigation to clarify the interactions of the microhabitat with external climate. Probably due to the alternating stages in their life cycles, protosteloid amoebae have the ability to resist changes in their environment. As each species can tolerate different types and ranges of change, individual abundances and species composition of the assemblages vary from locality to locality as the climate changes. These tendencies can be modelled and projected in maps of potential distribution, that constitute hypothesised probabilities of presence given a ubiquitous dispersal, and can be compared with actual presences. This could be a valuable tool in the future for unravelling biogeographic patterns and speciation processes.

\section{Acknowledgements}

We wish to thank Alejandro González for extracting values of environmental variables, Fátima Durán for technician work, and Diana Wrigley de Basanta for reviewing the text. This work was supported by the Research Projects CGL2005-00320/BOS and CGL2008-00720/BOS of the Ministry of Science and Innovation of Spain.

\section{References}

Agencia Estatal de Meteorología. (2011). Iberian Climate Atlas. Ministerio de Medio Ambiente y de Medio Rural y Marino: Madrid.

Aguilar M, Lado C, Spiegel FW. (2007). Protostelids from deciduous forests: first data from southwestern Europe. Mycol Res 111: 863-872.

Aguilar M, Spiegel FW, Lado C. (2011). Microhabitat and climatic preferences of protosteloid amoebae in a region with a mediterranean climate. Microb Ecol 62: 361-373.

Amato A, Kooistra WHCF, Levialdi Ghiron JH, Mann DG, Pröschold T, Montresor M. (2007). Reproductive isolation among sympatric cryptic species in marine diatoms. Protist 158: 193-207.

Anderson RP, Martínez-Meyer E. (2004). Modeling species' geographic distributions for preliminary conservation assessments: an implementation with the spiny pocket mice (Heteromys) of Ecuador. Biol Conser 116: 167-179.

Best SC, Spiegel FW. (1984). Protostelids and other simple mycetozoans of Hueston Woods State Park and Nature Preserve. In: Willeke GB (ed). Hueston Woods State Park and Nature Preserve, proceedings of a symposium, April 16-18. Miami University: Oxford, pp 116-121.

Brown MW, Spiegel FW. (2008). Assessment of protostelid diversity in Ozark Plateau oak-hickory forests in south central USA. In: Abstracts from 2007 MSA Meeting at LSU, Vol. 59; Inoculum, Baton Rouge, LA, p 9.

Di Castri F, Goodall DW, Specht RL. (1981). Ecosystems of the world II: Mediterranean- type shrublands. Elsevier Scientific Publishing Company: Amsterdam, Oxford, New York.

Douglas TE, Kronforst MR, Queller DC, Strassmann JE. (2011). Genetic diversity in the social amoeba Dictyostelium discoideum: Population differentiation and cryptic species. Molecular Phylogenetics and Evolution 60: $455-462$.

Ezard THG, Aze T, Pearson PN, Purvis A. (2011). Interplay between changing climate and species' ecology drives macroevolutionary dynamics. Science 332: 349-351.

Fenchel T, Finlay BJ. (2006). The diversity of microbes: resurgence of the phenotype. Phil Trans $R$ Soc Lond $B$ 361: 1956-1973.

Finlay BJ. (2002). Global dispersal of free-living microbial eukaryote species. Science 5570: 1061-1063.

Finlay BJ, Clarke KJ. (1999). Ubiquitous dispersal of microbial species. Nature 400: 828.

Finlay BJ, Esteban GF, Clarke KJ, Olmo JL. (2001). Biodiversity of terrestrial protozoa appears homogeneous across local and global spatial scales. Protist 152: 355-366. 
Finlay BJ, Esteban GF, Olmo JL, Tyler PA. (1999). Global distribution of free-living microbial species. Ecography 22: 138-144.

Finlay BJ, Fenchel T. (2004). Cosmopolitan metapopulations of free-living microbial eukaryotes. Protist 155: 237-244.

Foissner W. (2006). Biogeography and dispersal of microorganisms: a review emphasising protists. Acta Protozool 45: 111-136.

Foissner W, Chao A, Katz LA. (2008). Diversity and geographic distribution of ciliates (Protista: Ciliophora). Biodivers Conserv 17: 329-343.

Goslee S, Urban D. (2007). ecodist: Dissimilarity-based functions for ecological analysis. $\mathrm{R}$ package version 1.1.3.

Hijmans RJ, Cameron SE, Parra JL, Jones PG, Jarvis A. (2005). Very high resolution interpolated climate surfaces for global land areas. Int $J$ Climatol 25: 1965-1978.

Hutchinson GE. (1957). Concluding remarks. Cold Spring Harbor Symposia on Quantitative Biology 22: 415-427.

Kosheleva AP, Schnittler M, Novozhilov YK. (2009). Protostelids of the 'Stolby' State Reserve (Siberia, Eastern Sayan). Protistology 6: 24-32.

Moore DL, Spiegel FW. (1995). A new technique for sampling protostelids. Mycologia 87: 414-418.

Moore DL, Spiegel FW. (2000a). The effect of season on protostelid communities. Mycologia 92: 599-608.

Moore DL, Spiegel FW. (2000b). Microhabitat distribution of protostelids in temperate habitats in northwestern Arkansas. Can J Bot 78: 985-994.

Moore DL, Spiegel FW. (2000c). Microhabitat distribution of protostelids in tropical forests of the Caribbean National Forest, Puerto Rico. Mycologia 92: 616-625.

Moore DL, Stephenson S, Laursen G, Woodgate W. (2000). Protostelids from boreal forest and tundra ecosystems in Alaska. Mycologia 92: 390-393.

Moore DL, Stephenson SL. (2003). Microhabitat distribution of protostelids in a Tropical Wet Forest in Costa Rica. Mycologia 95: 11-18.

Morard R, Quillévéré F, Escarguel G, Ujiie Y, GaridelThoron T, Norris RD et al. (2009). Morphological recognition of cryptic species in the planktonic foraminifer Orbulina universa. Mar Micropaleontol 71: 148-165.

Ndiritu GG, Stephenson SL, Spiegel FW. (2009). First records and microhabitat assessment of protostelids in the Aberdare region, central Kenya. J Eukaryot Microbiol 56: 148-158.

Nychka D. (2007). Fields: tools for spatial data. R package version 4.1. http://www.image.ucar.edu/GSP/Software/ Fields.

Oksanen J, Kindt R, Legendre P, O’Hara B, Simpson GL, Stevens MHH et al. (2008). Vegan: community ecology package. R package version 1.13-1. http://vegan.r-forge. r-project.org/.

Olive LS. (1975). Chapter 2: Protostelia (Protostelids). In: Olive LS (eds). The Mycetozoans. Academic press: New York, pp 11-43.

Phillips SJ, Anderson RP, Schapire RE. (2006). Maximum entropy modelling of species geographic distributions. Ecol Model 190: 231-259.
Phillips SJ, Dudik M. (2008). Modeling of species distributions with Maxent: new extensions and a comprehensive evaluation. Ecography 31: 161-175.

Pianka ER. (1974). Niche overlap and diffuse competition. PNAS 71: 2141-2145.

Powers DM, Stephenson SL. (2006). Protostelids from tropical forests, woodlands and deserts in Australia. Mycologia 98: 218-222.

Pulliam HR. (2000). On the relationship between niche and distribution. Ecol Lett 3: 349-361.

R Development Core Team. (2008). R: a language and environment for statistical computing. R Foundation for Statistical Computing: Vienna, ISBN 3-900051-07-0, http://www.R-project.org.

Shadwick J, Stephenson S. (2004). First records of protostelids from northern India. Fungal Divers 16: 141-145.

Shadwick JDL, Stephenson SL, Spiegel FW. (2009a). Distribution and ecology of protostelids in Great Smoky Mountains National Park. Mycologia 101: 320-328.

Shadwick LL, Spiegel FW, Shadwick JDL, Brown MW, Silberman JD. (2009b). Eumycetozoa = Amoebozoa?: SSUrDNA phylogeny of protosteloid slime molds and its significance for the Amoebozoan supergroup. PLOS One 4: 1-13.

Smirnov AV. (2007). Cryptic freshwater amoeba species in the bottom sediments of Nivå Bay (Øresund, Baltic Sea). Eur J Protistol 43: 87-94.

Smith HG, Wilkinson DM. (2007). Not all free-living microorganisms have cosmopolitan distributions the case of Nebela (Apodera) vas Certes (Protozoa, Amoebozoa, Arcellinida). J Biogeogr 34: 1822-1831.

Spiegel FW. (1986). Phylum plasmodial slime molds class Protostelida. In: Margulis L et al. (eds). Handbook of Protoctista. Jones and Barlett: Boston, pp 484-497.

Spiegel FW, Shadwick JD, Lindley- Settlemyre L, Brown MW, Ndiritu G. (2007). A beginner's guide to identifying the protostelids, http://slimemold.uark.edu/pdfs/ Handbook1_3rd.pdf.

Spiegel FW, Stephenson SL. (2000). Protostelids of Macquarie Island. Mycologia 92: 849-852.

Spiegel FW, Stephenson SL, Keller HW, Moore DL, Cavender JC. (2004). Sampling the biodiversity of mycetozoans. In: Mueller GM et al (eds). Biodiversity of Fungi. Academic press: New York, pp 547-577.

Stephenson SL, Landolt JC, Moore DL. (1999). Protostelids, dictyostelids, and myxomycetes in the litter microhabitat of the Luquillo Experimental Forest, Puerto Rico. Mycol Res 103: 209-214.

Tesmer J, Rulik B, Spiegel F, Shadwick J, Schnittler M. (2005). Protostelids from German beech forests. Mycol Prog 4: 267-271.

Vanormelingen P, Verleyen E, Vyverman W. (2008). The diversity and distribution of diatoms: from cosmopolitanism to narrow endemism. Biodivers Conserv 17: 393-405.

Walter H. (1984). Vegetation of the earth in relation to climate and the ecophysiological conditions. The English Universities Press: London.

Weisse T. (2008). Distribution and diversity of aquatic protists: an evolutionary and ecological perspective. Biodivers Conserv 17: 243-259.

Supplementary Information accompanies the paper on The ISME Journal website (http://www.nature.com/ismej) 